\title{
Proteomic signatures corresponding to the SS18/SSX fusion gene in synovial sarcoma
}

\author{
Midori Ishii ${ }^{1, *}$, Yoshiyuki Suehara1,*, Kei Sano ${ }^{1}$, Shinji Kohsaka ${ }^{2}$, Takuo Hayashi ${ }^{3}$, \\ Saiko Kazuno ${ }^{4}$, Keisuke Akaike ${ }^{1}$, Kenta Mukaihara ${ }^{1}$, Youngji Kim ${ }^{1}$, Taketo Okubo ${ }^{1}$, \\ Kazuya Takamochi' ${ }^{5}$, Fumiyuki Takahashi ${ }^{6}$, Kazuo Kaneko ${ }^{2}$ and Tsuyoshi Saito ${ }^{3}$ \\ ${ }^{1}$ Department of Orthopedic Surgery, Juntendo University School of Medicine, Tokyo, Japan \\ ${ }^{2}$ Department of Medical Genomics, Graduate School of Medicine, The University of Tokyo, Tokyo, Japan \\ ${ }^{3}$ Department of Human Pathology, Juntendo University School of Medicine, Tokyo, Japan \\ ${ }^{4}$ Laboratory of Proteomics and Biomolecular Science, Research Support Center, Juntendo University School of Medicine, \\ Tokyo, Japan \\ ${ }^{5}$ Department of General Thoracic Surgery, Juntendo University School of Medicine, Tokyo, Japan \\ ${ }^{6}$ Department of Respiratory Medicine, Juntendo University School of Medicine, Tokyo, Japan \\ *These authors contributed equally to this work
}

Correspondence to: Yoshiyuki Suehara, email: ysuehara@juntendo.ac.jp

Keywords: synovial sarcoma, proteomics, SS18/SSX

Received: April 23, $2018 \quad$ Accepted: December 10, $2018 \quad$ Published: December 25, 2018

Copyright: Ishii et al. This is an open-access article distributed under the terms of the Creative Commons Attribution License 3.0 (CC BY 3.0), which permits unrestricted use, distribution, and reproduction in any medium, provided the original author and source are credited.

\section{ABSTRACT}

Synovial sarcoma (SS) is a malignant soft tissue lesion and most commonly arises in young adults. Chromosomal translocation $t(X ; 18)(p 11 ; q 11)$ results in the formation of SS18/SSX by gene fusion of the SS18 gene on chromosome 18 to either SSX1, SSX2, or SSX4 gene located on chromosome X, which is detected in more than $95 \%$ of SSs. Although multiple lines of evidence suggest that the SS18/SSX fusion is the oncogene in this tumor, the protein expression profiles associated with SS18/SSX have yet to be elucidated. In this study, we conducted proteomic studies using SS18/ SSX knockdown in three SS cell lines to identify the regulated proteins associated with SS18/SSX in SS. Isobaric tags for relative and absolute quantitation (i-TRAQ) analyses identified approximate 1700-2,000 proteins regulated by the SS18/SSX fusion in each SS cell line. We also analyzed the three profiles to identify proteins that were similarly altered in all 3 cell lines and found 17 consistently upregulated and 18 consistently downregulated proteins, including TAGLN and ACTN4. In addition, network analyses identified several critical pathways including RUNX2 and SMARCA4. RUNX2 and SMARCA4 had the highest ranking in these identified pathways. In addition, we found that expression of TAGLN inhibited cell viability in SS cell lines. Our data suggest that the differentiation and cell growth of SS may be enhanced by the identified proteins induced by SS18/SSX. We believe that the findings obtained in the present functional analyses will help to improve our understanding of the relationship between SS18/ SSX and malignant behavior in SS.

\section{INTRODUCTION}

Synovial sarcoma (SS) accounts for $7 \%-10 \%$ of all soft tissue malignancies and most commonly arises in the extremities of young adults with a slight male predominance $[1,2]$. SSs are high-grade sarcomas, since they lead to death in at least $25 \%$ of patients within the first 5 years after the diagnosis, despite advances in treatment [3]. Current therapeutic approaches are not particularly effective in the treatment of SSs, especially 
for patients with recurrence and metastasis [3, 4]. Therefore, a deeper knowledge of the genetic alterations and molecular mechanisms involved in SSs may allow for the development of novel therapeutic strategies. SS is characterized by recurrent chromosomal translocation of $\mathrm{t}(\mathrm{X} ; 18)(\mathrm{p} 11.2 ; \mathrm{q} 11.2)$, which results in the fusion of the $S S 18$ gene on chromosome 18 to either $S S X 1, S S X 2$ or SSX4 gene located on chromosome $\mathrm{X}$, giving the fusion protein product SS18/SSX [5-8]. In addition, an analogous translocation of $S S X 4$ is detected in less than $1 \%$ of cases $[9,8]$. Multiple lines of evidence have suggested that the SS18/SSX fusion is an oncogene and the central genetic "driver" in SS; (i) its presence as a sole cytogenetic anomaly in up to one-third of cases [9, 10], (ii) the low frequency of additional mutations [9, 11], (iii) its preservation in metastatic and advanced lesions $[9,10]$, (iv) the death of synovial sarcoma cells upon SS18/SSX knockdown [9, 12], and (v) its ability to induce tumors in conditional mouse models with appropriate histology, gene expression, and immunophenotype with $100 \%$ penetrance $[9,13]$. In particular, the expression of INI1/SMARCB1/BAF47 is low or absent, while that of NY-ESO-1, a cancer-testis antigen, is high in SSs, as previously mentioned [14-16].

Analyzing the SS18-SSX function has been difficult, given the lack of an appropriate model system as the origin cells of SS are unknown [17]. Furthermore, most studies on SS have relied on heterologous cell types of uncertain relevance to human disease, with findings suggesting that the expression of genes associated with SS18/SSX may differ by cell type [17-20]. Given that the cellular background is critical for determining the function of SS18/SSX fusion protein in human SS, gene expression studies have employed SS18-SSX silencing by siRNA using three SS cell lines and identified genes related to SS18/SSX and several candidates for novel molecular therapy $[2,17]$. However, the protein expression profiles associated with SS18/SSX have yet to be elucidated.

In this study, to identify the regulated proteins associated with SS18/SSX and clarify the function of SS18/SSX, we conducted proteomic studies using SS18/ SSX knockdown in SS cell lines. We also conducted network analyses based on the protein profiles and carried out functional analyses of an identified critical protein and pathway.

\section{RESULTS}

\section{SS18/SSX knockdown in SS cell lines}

SS18/SSX knockdown was performed using three siRNA designed for the $S S 18 / S S X$ break point $(S S 18 / S S X$ BP_A, B and C). The SS18/SSX BP_A siRNA showed the strongest inhibition among these siRNAs in HS-SYII (Supplementary Figure 1). The SS18/SSX BP_A siRNA inhibited the SS18/SSX expression in all three SS cell lines, including the HS-SYII, YaFuSS, and SYO-1 lines (Figure 1 and Supplementary Figure 1). Regarding the cell proliferation, SS18/SSX knockdown with these siRNAs efficiently suppressed the cell proliferation of HS-SYII, YaFuSS, and SYO-1. We selected SS18/SSX BP A siRNA to use in the subsequent experiments, as this siRNA showed the strongest effect.

\section{Identification of protein profiles associated with SS18/SSX expression in SS cell lines by i-TRAQ}

To identify protein profiles associated with SS18/ SSX, we performed i-TRAQ analyses using SS cells (HS-SYII, YaFuSS and SYO-1) transfected with siRNA against the $S S 18 / S S X$ break point (SS18/SSX BP_A) or control. Proteins were extracted from both transfected SS cells. i-TRAQ analyses using each cell were carried out and identified 1976 (HS-SY II), 1976 (YaFuSS) and 1784 (SYO-1) or 1700-2000 proteins in each analysis. A statistical comparison led to the compilation of the protein profile, which differed significantly between the siRNA targets and control. In the SS cells with knockdown of SS18/SSX (Figure 1 and Supplementary Tables 1-3), analyses showed 33 downregulated proteins associated with SS18/SSX knockdown in HS-SYII, 36 in YaFuSS, and 34 in SYO-1 $(p<0.05)$. Furthermore, analyses showed 72 upregulated proteins associated with $S S 18 / S S X$ knockdown in HS-SYII, 44 in YaFuSS, and 46 in SYO-1 $(p<0.05)$. We analyzed the 3 profiles to identify proteins that were similarly altered in all 3 cell lines and found 18 consistently upregulated and 17 consistently downregulated proteins, including TAGLN and ACTN4 (Table 1, Supplementary Figure 2 and Supplementary Figure 3).

\section{Network analyses according to the protein profiles of SS18/SSX}

To further understand the biological processes and networks involved in tumor malignancy based on the proteins regulated by $S S 18 / S S X$, we performed network analyses using the Ingenuity Pathways Analysis (IPA) system (QIAGEN, Redwood City, CA, USA). We conducted analyses using the proteins associated with $S S 18 / S S X$ knockdown in SS cell lines. In the network analyses based on the profiles of $S S 18 / S S X$ knockdown in SS cells, we identified several pathways involving RUNX2 and SMARCA4 that played a critical functional role and acted as upstream regulators of these proteins known to be associated with the SYT/SSX expression in SS cells (Supplementary Tables 4 and Table 5).

\section{Confirmation of the mRNA expression in identified proteins and pathways}

To confirm the mRNA expression corresponding to the identified proteins by proteomic analyses (i-TRAQ), 
we conducted qPCR of TAGLN and ACTN4 using three SS cell lines (HS-SYII, YaFuSS, and SYO-1) that were transfected with $S S 18 / S S X$ siRNA (siRNA SS18/SSX BP_A). Regarding $T A G L N$, the cell lines subjected to siRNA SS18/SSX knockdown showed significantly higher TAGLN expression than control samples (Figure 2A). Regarding ACTN4, the cell lines subjected to siRNA SS18/ $S S X$ knockdown had significantly higher mRNA levels of ACTN4 than control samples (Figure 2B). These results suggest that our protein profiles for SS18/SSX were accurate.

With respect to IPA analyses, we identified several critical pathways including RUNX2 and CDKN2A. To confirm whether these findings matched the expression in SS, we investigated the mRNA status of both $R U N X 2$ and $C D K N 2 A$ in SS18/SSX-regulated SS cells. The results showed that the expression of $R U N X 2$ and $C D K N 2 A$ were consistently higher in all $S S 18 / S S X$-silenced SS cell lines than in control samples (Supplementary Figure 4). These results indicated that our protein profiles and pathway analyses had correctly identified proteins and transcript pathways that were regulated by SS18/SSX fusion.

\section{Effects of the TAGLN expression on the proliferation of SS cells}

To investigate the association between TAGLN and the proliferation of SS cells, TAGLN forced expression was performed via TAGLN transfection in a SS cell line (SYO-1). q-PCR revealed that TAGLN-transfected cells had significantly higher TAGLN expression than control cells (GFP transfected cells) in the SYO-1 cell line (Figure 3A). In the cell proliferation assays, we also found that the cell proliferation was suppressed in SS cell lines by TAGLN expression (Figure 3B). These results revealed that silencing SS18/SSX suppressed SS cell growth in part via activation of TAGLN expression. Therefore, based on these findings, we concluded that our protein profiles contained crucial information regarding tumor malignancy and potential therapeutic targets in SS cells.

\section{DISCUSSION}

Metabolic or chemical labeling was used for the quantitative proteomic analyses by mass spectrometry.

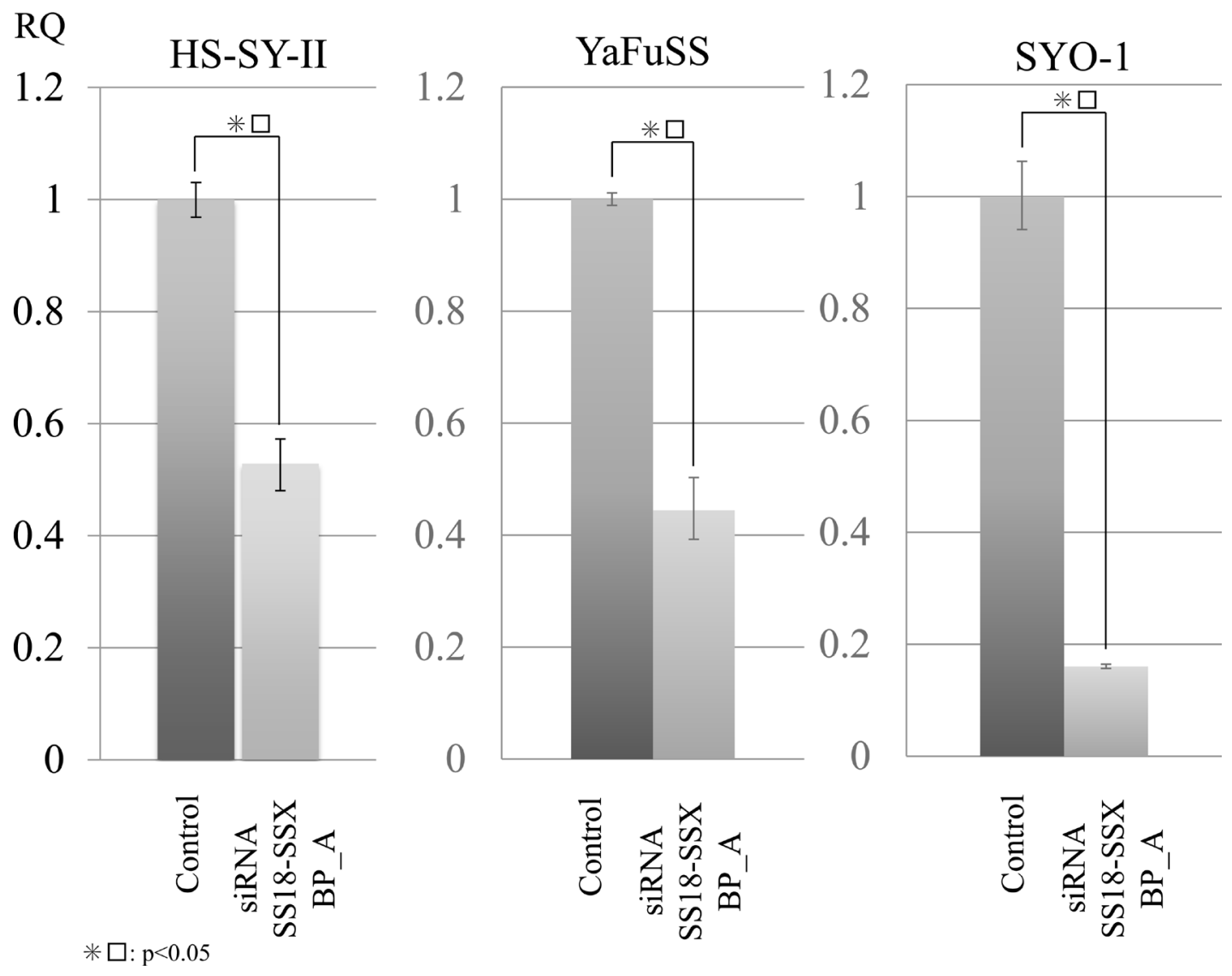

Figure 1: Proteomic analyses of SS18/SSX using SS18/SSX break point siRNA inhibited SS cell lines. To identify protein profiles regulated by SS18/SSX, we performed i-TRAQ analyses using SS cells (HS-SYII, YaFuSS and SYO-1) transfected with siRNA against the SS18/SSX break point (SS18/SSX BP_A) or control. Before the proteomic analyses, we performed siRNA assays to generate these proteins. Quantitative PCR (qPCR) assays showed that siRNA SS18/SSX BP inhibited the mRNA expression of SS18/SSX in all three SS cell lines. 
Table 1: Proteins regulated by the SS18/SSX fusion gene

\begin{tabular}{|c|c|c|c|c|}
\hline Accession no. & Symbol & Protein name & Fold difference & $P$ value \\
\hline Q01995 & TAGL_HUMAN & Transgelin & 2.32 & $0.00 \mathrm{E}+00$ \\
\hline P09493-3 & TPM1_HUMAN & Isoform 3 of Tropomyosin alpha-1 chain & 2.02 & $3.20 \mathrm{E}-03$ \\
\hline P06703 & S10A6_HUMAN & Protein S100-A6 & 1.87 & $3.02 \mathrm{E}-02$ \\
\hline P04792 & HSPB1_HUMAN & Heat shock protein beta- 1 & 1.65 & $1.88 \mathrm{E}-02$ \\
\hline O43707 & ACTN4_HUMAN & Alpha-actinin-4 & 1.49 & $2.80 \mathrm{E}-03$ \\
\hline P51884 & LUM_HUMAN & Lumican & 1.41 & $1.67 \mathrm{E}-02$ \\
\hline P67936 & TPM4_HUMAN & Tropomyosin alpha-4 chain & 1.34 & $2.00 \mathrm{E}-02$ \\
\hline P49902-2 & 5NTC_HUMAN & Isoform 2 of Cytosolic purine 5'-nucleotidase & 1.31 & $3.70 \mathrm{E}-02$ \\
\hline P21333-2 & FLNA_HUMAN & Isoform 2 of Filamin-A & 1.29 & $1.33 \mathrm{E}-04$ \\
\hline P08758 & ANXA5_HUMAN & Annexin A5 & 1.29 & $2.50 \mathrm{E}-04$ \\
\hline Q14315 & FLNC_HUMAN & Filamin-C & 1.25 & $1.70 \mathrm{E}-03$ \\
\hline P35579 & MYH9_HUMAN & Myosin-9 & 1.24 & $0.00 \mathrm{E}+00$ \\
\hline Q99439 & CNN2_HUMAN & Calponin-2 & 1.24 & $8.90 \mathrm{E}-03$ \\
\hline P27797 & CALR_HUMAN & Calreticulin & 1.23 & $1.40 \mathrm{E}-03$ \\
\hline P07237 & PDIA1_HUMAN & Protein disulfide-isomerase & 1.23 & $1.53 \mathrm{E}-03$ \\
\hline P18206 & VINC_HUMAN & Vinculin & 1.22 & $2.10 \mathrm{E}-03$ \\
\hline P29401 & TKT_HUMAN & Transketolase & 1.14 & $1.40 \mathrm{E}-02$ \\
\hline P23284 & PPIB_HUMAN & Peptidyl-prolyl cis-trans isomerase B & 1.13 & $2.52 \mathrm{E}-02$ \\
\hline P31948 & STIP1_HUMAN & Stress-induced-phosphoprotein 1 & 0.90 & $9.75 \mathrm{E}-03$ \\
\hline P50990 & TCPQ_HUMAN & T-complex protein 1 subunit theta & 0.89 & $1.61 \mathrm{E}-02$ \\
\hline P49327 & FAS_HUMAN & Fatty acid synthase & 0.88 & $3.70 \mathrm{E}-03$ \\
\hline P48643 & TCPE_HUMAN & T-complex protein 1 subunit epsilon & 0.88 & $7.43 \mathrm{E}-03$ \\
\hline P17987 & TCPA_HUMAN & T-complex protein 1 subunit alpha & 0.87 & $3.17 \mathrm{E}-02$ \\
\hline P78371 & TCPB_HUMAN & T-complex protein 1 subunit beta & 0.87 & $5.25 \mathrm{E}-03$ \\
\hline P40227 & TCPZ_HUMAN & T-complex protein 1 subunit zeta & 0.87 & $2.10 \mathrm{E}-02$ \\
\hline P09429 & HMGB1_HUMAN & High mobility group protein $\mathrm{B} 1$ & 0.86 & $1.48 \mathrm{E}-02$ \\
\hline P39023 & RL3_HUMAN & 60 S ribosomal protein L3 & 0.84 & $6.80 \mathrm{E}-03$ \\
\hline P08238 & HS90B_HUMAN & Heat shock protein HSP 90-beta & 0.83 & $1.73 \mathrm{E}-02$ \\
\hline P53396 & ACLY_HUMAN & ATP-citrate synthase & 0.82 & $1.92 \mathrm{E}-02$ \\
\hline P40429 & RL13A_HUMAN & $60 \mathrm{~S}$ ribosomal protein $\mathrm{L} 13 \mathrm{a}$ & 0.82 & $1.35 \mathrm{E}-02$ \\
\hline P50454 & SERPH_HUMAN & Serpin H1 & 0.81 & $7.55 \mathrm{E}-03$ \\
\hline P05141 & ADT2_HUMAN & ADP/ATP translocase 2 & 0.79 & $2.15 \mathrm{E}-03$ \\
\hline P26641 & EF1G_HUMAN & Elongation factor 1-gamma & 0.78 & $2.75 \mathrm{E}-03$ \\
\hline P62750 & RL23A_HUMAN & $60 \mathrm{~S}$ ribosomal protein $\mathrm{L} 23 \mathrm{a}$ & 0.76 & $2.55 \mathrm{E}-02$ \\
\hline P62917 & RL8_HUMAN & $60 \mathrm{~S}$ ribosomal protein L8 & 0.75 & $2.44 \mathrm{E}-02$ \\
\hline
\end{tabular}

Stable isotope labeling using amino acids in cell culture (SIALC) uses metabolic labeling and is applied only to cultured cells. In contrast, isobaric tag for relative and absolute quantitation (iTRAQ) uses chemical labeling and is able to label both biological samples and cultured cells with high efficiency. Regarding the quantification ability, both methods provide accurate results [21].

We conducted a proteomic study (iTRAQ) focusing on SS18/SSX fusion in SS and successfully identified several characteristic proteins as being associated with the SS18/SSX expression. To our knowledge, this study is the first report regarding the protein expression of SS18/SSX fusion in SS. Our protein profiles consistently identified 35 proteins regulated by the SS18/SSX expression across 3 SS cell lines. In these protein profiles obtained by the silencing of SS18/SSX fusion, 18 upregulated proteins that had the opposite expression to SS18/SSX and 17 downregulated proteins that had synchronous expression with SS18/SSX were identified.

Regarding to accuracy of our proteomic study, Heat shock protein (HSP) 90-beta and tropomyosin alpha 4 chain were also identified in both our SS18/SSX and SS 
surgical material studies [22]. HSP 90-beta was expressed in the group with a poor prognosis in the SS surgical material study and is listed among the proteins downregulated by SS18/SSX. Tropomyosin alpha 4 chain was expressed in the group with a good prognosis in the SS surgical material study and is listed among the proteins up-regulated by SS18/SSX. These expression trends in these two proteins were coincident with both the surgical material and fusion gene profiles, suggesting that our protein profiles for SS18/SSX were accurate.

Among the 18 upregulated proteins, we detected several smooth muscle proteins, including transgelin (TAGLN), tropomyosin alpha-1 chain (TPM1), tropomyosin alpha-4 chain (TPM4), myosin-9 (MYH9), and calponin-2 (CNN2). TAGLN demonstrated the highest fold changes in our protein profile regulated by silencing SS18/SSX. A previous report found that TAGLN was specifically expressed in 154 of 184 leiomyosarcoma (LMS) (84\%) and could be used as a novel diagnosis marker for tumor of smooth muscle differentiation, especially for LMS [23]. The high expressions of tropomyosin, myosin and calponin families have also been mentioned in proteomic expression studies as LMS specifically [24, 25]. Base on the previous studies and these identified proteins regarding SS18/SSX, our novel proteomic profile seems to indicate that SS18/ SSX might have the ability to interrupt smooth muscle differentiation.

The cellular origin and differentiation of SS remain still unclear, but both mesenchymal and epithelial differentiation have been observed [26-28]. In gene expression studies, the high expression of neural lineage makers, including NPTX2, NEFH, and NTNG2, as well as chondrocyte lineage markers, including COL2A1, COL9A3, SOX9, and TRPS1, have been interpreted as consistent with differentiation from neural crest progenitors [9]. The high mRNA expression of $S O X 2$ also functions as a critical determinant of neural progenitors and embryonic stem cells in SS [9, 29, 30]. Several recent reports have suggested that SS is most likely derived from primitive mesenchymal cells that undergo differentiation [30-33]. In addition, regarding the primitive origin of $\mathrm{SS}$, the expression of human SS18-SSX2 fusion protein within myoblasts in transgenic mouse model was shown to produce tumors similar to human SSs $[31,13,34]$. These
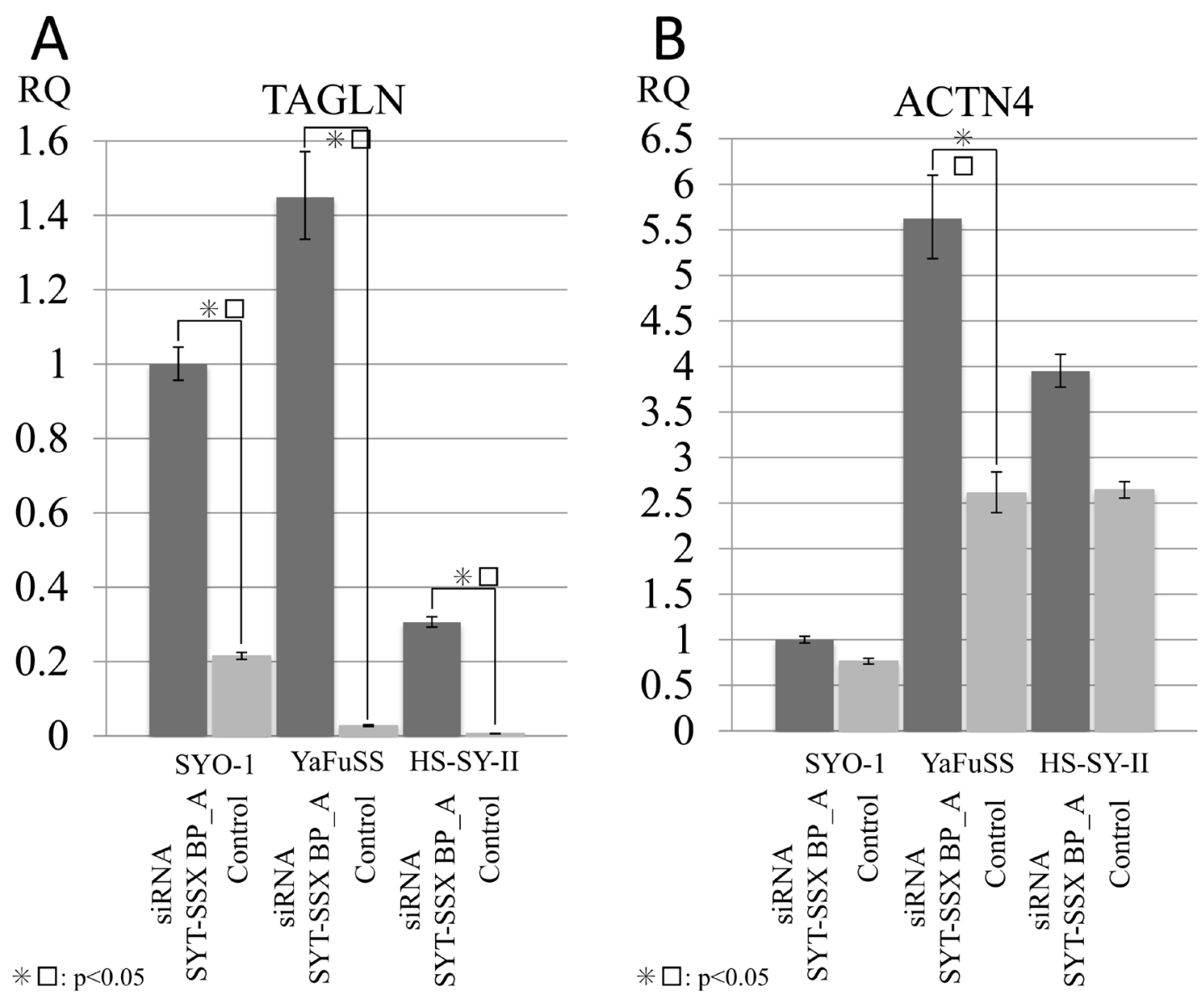

Figure 2: Silencing SS18/SSX activated the expression of TAGLN and ACTN4. To investigate the association of SS18/SSX with TAGLN and ACTN4, siRNA SS18/SSX was performed, and the mRNA expression was measured by q-PCR. The silencing of P SS18/ SSX activated the expression of both TAGLN (A) and ACT4 (B) in SS cell lines (HS-SYII, YaFuSS, and SYO-1). 
present and previous findings suggest that SS18/SSX might suppress smooth muscle differentiation, while offering clues to the cellular origin and real differentiation of SS. In addition, in our confirmation studies using qPCR, silencing of SS18/SSX in SS activated the mRNA expression of TAGLN, which suppressed cell proliferation in SS cell lines. These findings suggested that our protein profiles also provide clues associated with tumor malignancy and identified several candidate therapeutic targets.

Gene expression studies regarding the regulators of SS18/SSX have identified several interesting genes $[35,12,2,17]$. Takenaka et al. found that ACTN4, which our protein profiling also identified as upregulated by silencing SS18/SSX, reduced the malignant potential in SS [17]. ACTN4 performs crucial roles in signaling transduction, nuclear translocation, and gene expression regulation [36]. Honda et al. reported that ACTN4 contributed to cell motility and was highly expressed in breast cancer. In addition, ACTN4 is found in the membrane ruffles, and the inhibition of PI3 kinase (PI3K) promotes ACTN4 nuclear translocation in breast cancer and several cancer cell lines $[37,38]$. These findings indicate that ACTN4 is closely associated with malignant potential in several cancers; however, interestingly, our proteomic study and previous gene expression studies have obtained the opposite findings in SS, namely showing that ACTN4 has the ability to suppress malignancy. Takenaka et al. also performed several functional studies focusing on adhesion including ACTN4 and ultimately concluded that SS18-SSX1 enhances adhesion to the extracellular matrix through the induction of expression of myosin light-chain kinase [17]. Our proteomic studies also identified myosin-9 as the myosin family. These findings might provide crucial information for clarifying the differences in the malignant behavior between epithelial cancers and SS. In addition, while other gene expression studies on silencing SS18/SSX in SS cells identified COM1 as a critical gene regulated by SS18/SSX and functioning as a cell proliferation regulator in SS, our protein profiling didn't identify show that COM1 was such an abundant protein [35].

We also performed pathway analyses using the IPA software program and identified several crucial pathways, including runt-related transcription factor 2 (RUNX2) and SMARCA4. RUNX2 is also known as core-binding factor
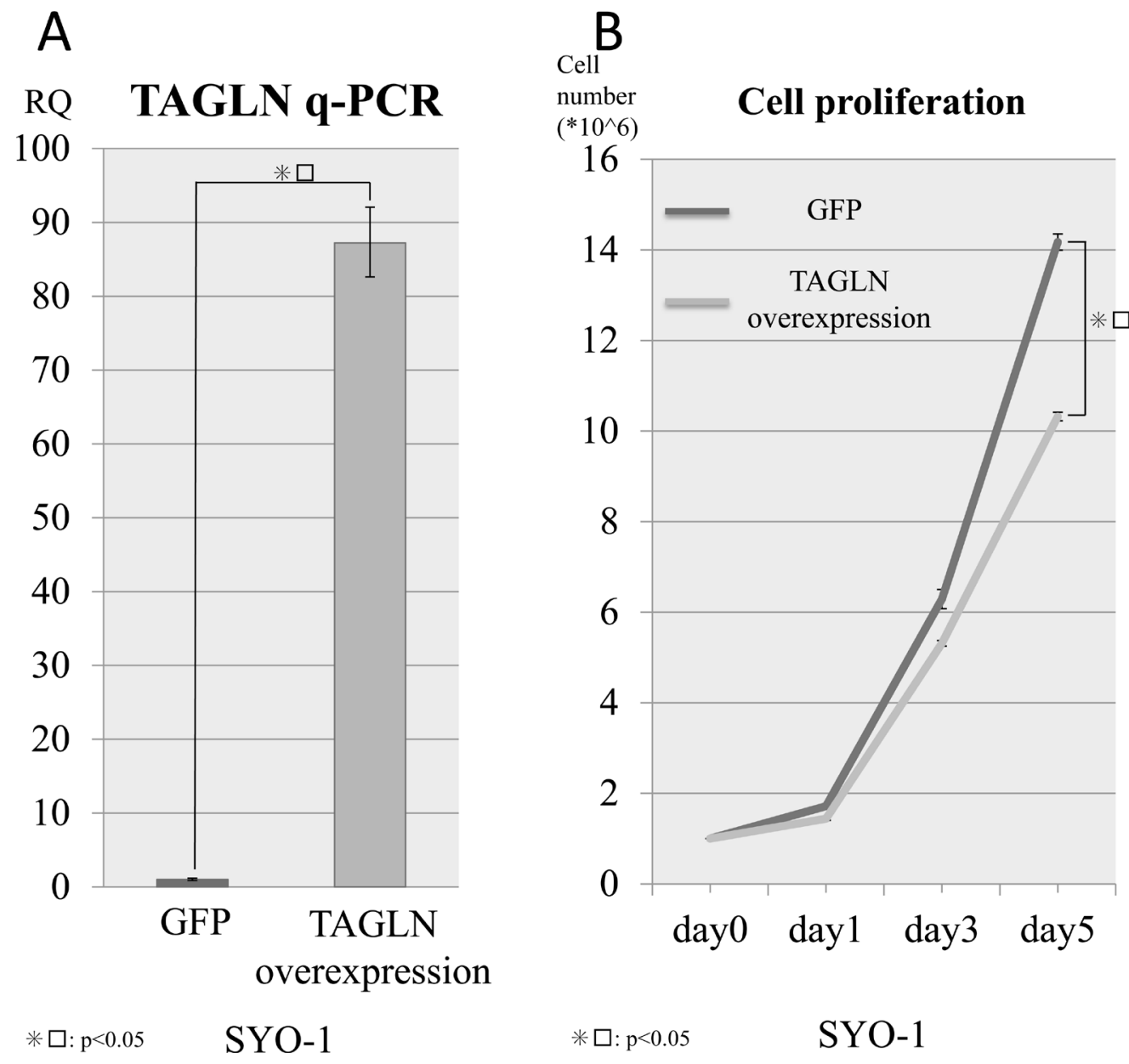

Figure 3: Cell viability with TAGLN overexpression. TAGLN was transfected in SYO1 cells lines via retrovirus transduction to verify the associations between the TAGLN expression and cell viability. (A) q-PCR confirmed the overexpression of TAGLN in the SYO cell line and (B) the expression of TAGLN inhibited the cell viability in the SYO-1 cell line compared with control samples (GFP). 
subunit alpha-1 (CBF-alpha-1) and is a key transcription factor associated with osteoblast differentiation in humans [39]. The radiologic characteristic of SS, found in $15 \%-20 \%$ of cases, is the presence of multiple small, spotty radiopacities caused by focal calcification and less frequently bone formation [1]. Of note, some reports have revealed that SSs with extensive calcification are associated with a good prognosis, and such calcifying SS cases have shown a 5-year survival rate of $82 \%$ $[1,40]$. Furthermore, several histopathogenesis studies have explored the expression of RUNX2 in SS, and our finding of RUNX2 in the protein profile of SS was in accordance with the findings of those previous studies $[1,41,42]$. However, the histological effect of the RUNX2 expression on metaplastic calcification in SS is unclear, as SS18/SSX seems to suppress the RUNX2 expression in SS based on the data from these studies. Regarding SMARCA4, the SWI/SNF (BAF) complex includes SMARCA4, and the epigenetic modification of SS18/SSX alters the chromatin remodeling via epigenetic alterations through SWI/SNF(BAF)- and histone deacetylase (HDAC)-associated mechanisms [9, 43, 26, 44-47]. Recently, Kadoch described the high-affinity binding of SS18/SSX fusion to the core subunits of the SWI/SNF (BAF) complex and SS18 and SS18/SSX incorporate into the core subunits of SWI/SNF (BAF) complex, by dissociating BAF47 from the complex, thereby releasing SNF5, a tumor suppressor [43].

Other proteomic studies have recently been carried out for soft tissue sarcomas with specific fusion genes such as EWS/FLI1 (for Ewing sarcomas) and PAX3/ FOXO1 (for alveolar rhabdomyosarcoma). These studies have identified numerous proteins; 90 proteins for EWS/ FLI1 and 221 proteins for PAX3/FOXO1, despite this SS study have just identified 35 proteins for SS18/SSX $[48,49]$. Several functional studies have found that SS18/SSX is not a transcriptional factor but co-activator [1, 9, 43, 26, 44-47]. In contrast, EWS/FLI1 and PAX3/ FOXO1 were described as critical transcriptional factors in numerous previous studies [48, 49]. Therefore, based on these functional backgrounds, silencing of coactivator SS18/SSX picked up small amounts of regulated proteins compared to Ewing sarcoma and alveolar rhabdomyosarcoma having either EWS/FLI1 or PAX3/ FOXO1 which act as transcriptional factors. With respect to differences in the expression of proteins that were regulated by these fusion genes, the characteristic genome status of SS18/SSX, a co-activator, might highlight slight differences in the expression of proteins regulated by SS18/SSSX compared to transcript factors (EWS/FLI1 and FOX3/FOXO1) [48, 49].

In the present study, we conducted proteomic analyses by silencing $S S 18 / S S X$ in SS cells. Our data suggest that the differentiation and cell growth of SS may be enhanced by the identified proteins induced by SS18/ SSX. We believe that our established protein profiles will help to improve the understanding of the relationship between SS18/SSX and the oncogenic behavior in SS, thereby leading to the development of novel therapeutic strategies.

\section{MATERIALS AND METHODS}

\section{Cell lines}

Three synovial sarcoma cell lines of HS-SY-II (Dr. Hiroshi Sonobe, Kochi University, Nankoku, Japan), YaFuSS (Dr. Junya Toguchida, Kyoto University, Kyoto, Japan), and SYO1 (Dr. Akira Kawai, Okayama University, Okayama, Japan) were kindly provided. All cell lines were grown in Dulbecco's modified Eagle's medium (DMEM) supplemented with $10 \% \mathrm{FCS}$ and penicillin/streptomycin.

\section{RNA extraction, quantitative real-time PCR (q-PCR) and reverse transcription-polymerase chain reaction (RT-PCR)}

RNA was isolated using an RNeasy Plus Mini kit (Qiagen, Hilden, Germany), and first-strand synthesis was performed using $5 \mu \mathrm{g}$ of RNA and the SuperScript ${ }^{\circledR}$ IV First-Strand Synthesis System (Thermo Fisher Scientific, Commonwealth, MA, USA). Quantitative real-time PCR (qPCR) was performed using inventoried Taqman assays from Applied Biosystems (Carlsbad, CA, USA) (20× Primer Probe mix) corresponding to SS18/SSX1 (Assay ID Hs03024820_ft), TAGLN (Assay ID Hs01038777_g1), ACTN4 (Assay ID Hs00245168_m1), RUNX2 (Assay ID Hs00231692_m1), CDKN2A (Assay ID Hs00923894 m1), ACTB (Assay ID Hs01060665_g1), and GAPDH (Assay ID Hs02758991_g1). All PCR reactions were performed with a TaqMan Fast Advanced Master Mix (Applied Biosystems) on an Applied Biosystems Step One Plus Real Time PCR System in accordance with the standard protocols. The amount of each target gene relative to the housekeeping gene ACTB and GAPDH was determined using the comparative threshold cycle (Ct) method. For each sample, the relative amount of each target gene was calibrated against a control transfected cell line. All assays were performed in triplicate. RTPCR analyses were carried out to evaluate the expression of SS18/SSX using PCR SuperMix (Thermo Fisher Scientific). The human SS18/SSX primer sequences were as follows: 5'-CAGCAGAGGCCTTATGGATATGA-3' and 5'-TTTGTGGGCCAGATGCTTC-3'. GAPDH was used as a loading control with the following primers: 5'-GAAGGTGAAGGTCGGAGTC3' and 5'- GAAGATGGTGATGGGATTT-3'.

\section{SS18/SSX siRNA in SS cell lines}

For the protein expression studies based on knockdown system, we used three cell lines (HS-SY-II, 
YaFuSS and SYO1). SS cell lines expressing SS18/SSX were treated with $50 \mathrm{nM}$ of SS18/SSX break-point siRNA for A: Sense 5'-CCAGAUCAUGCCCAAGAAGdTdT-3' Antisense 3'-dTdTGGUCUAGUACGGGUUCUUC-5', B: Sense 5'-CCAGAUCAUGCCCAAGAAdTdT-3', Antisense 3'-dTdTGGUCUAGUACGGGUUCUU-5', C: Sense 5'-GACCAGAUCAUGCCCAAGAUU-3', Antisense 3'-UUCUGGUCUAGUACGGGUUCU-5', using Lipofectamine ${ }^{\mathrm{TM}}$ RNAiMAX reagent (Thermo Fisher Scientific). After $72 \mathrm{~h}$, the total protein and RNA from each cell line was isolated, and the expression was validated by qPCR and reverse RT-PCR.

\section{Western blotting}

The proteins were extracted from four SS cells, separated via SDS-PAGE and transferred to nitrocellulose membranes. The membranes were then incubated with either of the following antibodies: rabbit monoclonal antibodies against SS18/SSX (\#70929, dilution 1:200; Cell Signaling, Danvers, MA, USA) or mouse monoclonal antibody against GAPDH (\#sc-32233, dilution 1: 500; Santa Cruz, Dallas, TX, USA). After incubation, the membranes were washed 3 times with Tris-EDTA buffer and then reacted with horseradish peroxidase-conjugated secondary antibodies (dilution 1:1,000; GE Healthcare Biosciences, Boston MA).

\section{Isobaric tags for relative and absolute quantification (i-TRAQ) sample labeling, mass spectrometry analyses, and peptide identification}

Analyses of proteins by i-TRAQ, a chemical label detected by mass spectrometry, were performed as described in our previous article (RR, SS). In brief, cell lysate samples was concentrated and buffer-exchanged using 3.5-kDa molecular weight cut-off spin concentrators (Tomy Seiko Co., Ltd., Tokyo, Japan) and then digested overnight with $10 \mu \mathrm{g}$ L-1-(4-tosylamido)-2-phenylethyl tosylphenylalanyl chloromethyl ketone-treated trypsin. Each peptide solution was labeled with 1 of the 8 iTRAQ reagents (iTRAQ reporter ions of 113, 114, 115, 116, 117, 118,119 , and 121 mass/charge ratio) in accordance with the manufacturer's protocol (AB SCIEX, Framingham, MA, USA). Labeled peptides were pooled and fractionated by strong cation exchange to four fractions. Each fraction was desalted with Sep-Pak C18 Plus Light Cartridge (Waters Corporation, Milford, MA, USA) and analyzed using nano liquid chromatography coupled with tandem mass spectrometry (LC-MS/MS); nano LC-MS was performed on a TripleTOF 5600 mass spectrometer for MS/MS operated with the Analyst TF 1.7 software program (AB SCIEX, Dublin, CA, USA) interfaced with the Eksigent nano LC system using a ChromXP C18-CL column (AB SCIEX). Protein identification and relative quantification was carried out using the ProteinPilot software program, version 5.0 (AB SCIEX). The functions of the various protein contents were determined by searching the UniProt database using the search algorithm within the ProteinPilot program (AB SCIEX). Protein ratios were normalized using the overall median ratio for all peptides in the sample for each separate ratio in every individual experiment. Two independent iTRAQ experiments were carried out to profile and quantitate the proteome, and the three technical replicates were used to determine the cut-off for significant fold-changes. A confidence cut-off of $>95 \%$ was applied for protein identification, and a $>1.2$-fold change cut-off for all iTRAQ ratios was selected to classify proteins as up- or downregulated.

\section{Pathway analyses}

The pathway analysis is shown in our previous article [50, 48]. In brief, the IPA software program (Qiagen) was further employed to determine the functional pathways of the identified genes. The IPA software program contains a database of biological interactions among genes and proteins and was used to calculate the probability of relationships among each of the canonical pathways, the upstream pathways, and the identified proteins. The IPA program scans the proteins that are entered by the user in order to identify networks using the Ingenuity Pathway Knowledge Base (IPKB) based on interactions between identified proteins and the known and hypothetical interacting genes stored in the program. The IPA program has been licensed for free use since 2013.

\section{Preparation of retrovirus and transduction of cell lines}

For retrovirus production, the pcx4 (PMID: 14597713) and pGEM (Promega) vector systems were used. A plasmid encoding human TAGLN (Origene) was used to generate constructs, which were subcloned into pGEM, and the construct were subcloned into pCX4bleo. Retroviruses were obtained using $\alpha \varphi$ cells as packaging cells and infected into $3 \mathrm{SS}$ lines for selection with $500 \mu \mathrm{g} / \mathrm{ml}$ zeocin (Thermo Fisher Scientific).

\section{Cell proliferation with TAGLN overexpression}

To evaluate cell viabilities with the over-expression of TAGLN in SS, $1.0 \times 10^{6} \mathrm{SS}$ cells were plated into $100 \mathrm{~mm}$ dishes on day 0 with control (GFP). The cells were counted at $24 \mathrm{~h}, 72 \mathrm{~h}$, and $120 \mathrm{~h}$, and all proliferation experiments were performed in triplicate, with the results averaged.

\section{Statistical analyses}

The relationships between the protein expression, cell proliferation, and other factors were analyzed using Fisher's exact test or a $t$-test. 


\section{ACKNOWLEDGMENTS AND FUNDING}

This study was supported by a Grant-in-Aid from the Japan Society for the Promotion of Science (JSPS) KAKENHI (Grant Number \#15H04964, \#16K15670, and \#15KK0353 to Y.S.; \#17K08730 to T.S.; \#17K10987to K.K.; 17H07098 to K.A.; \#16K20070 to T.O.; \#16H07186 to K.M.) and Leading Advanced Projects for Medical Innovation (LEAP) (Grant Number \# JP17am0001009) from the Japan Agency for Medical Research and Development. The authors thank Dr. Hiroshi Sonobe (Kochi University, Nankoku, Japan), Dr. Junya Toguchida (Kyoto University, Kyoto, Japan), and Dr. Akira Kawai (Okayama university, Okayama, Japan) for kindly providing the SS cell lines.

\section{CONFLICTS OF INTEREST}

The authors declare no conflicts of interest in association with this study.

\section{REFERENCES}

1. Oliveira AM. Enzinger and Weiss's Soft Tissue Tumors. 4th edn. 2001.

2. Peng C, Guo W, Yang Y, Zhao H. Downregulation of SS18SSX1 expression by small interfering RNA inhibits growth and induces apoptosis in human synovial sarcoma cell line HS-SY-II in vitro. Eur J Cancer Prev. 2008; 17:392-8. https://doi.org/10.1097/CEJ.0b013e328305a11b.

3. Lewis JJ, Antonescu CR, Leung DH, Blumberg D, Healey JH, Woodruff JM, Brennan MF. Synovial sarcoma: a multivariate analysis of prognostic factors in 112 patients with primary localized tumors of the extremity. J Clin Oncol. 2000; 18:2087-94. https://doi.org/10.1200/ jco.2000.18.10.2087.

4. Ly JQ, LaGatta LM, Beall DP, Packard J. Massive recurrent synovial sarcoma of the hip: radiologichistologic correlation in a case of failed magnet therapy. Clin Imaging. 2005; 29:291-3. https://doi.org/10.1016/ s0899-7071(03)00016-0.

5. Clark J, Rocques PJ, Crew AJ, Gill S, Shipley J, Chan AM, Gusterson BA, Cooper CS. Identification of novel genes, SYT and SSX, involved in the $\mathrm{t}(\mathrm{X} ; 18)(\mathrm{p} 11.2 ; \mathrm{q} 11.2)$ translocation found in human synovial sarcoma. Nat Genet. 1994; 7:502-8. https://doi.org/10.1038/ng0894-502.

6. Smith S, Reeves BR, Wong L, Fisher C. A consistent chromosome translocation in synovial sarcoma. Cancer Genet Cytogenet. 1987; 26:179-80.

7. Crew AJ, Clark J, Fisher C, Gill S, Grimer R, Chand A, Shipley J, Gusterson BA, Cooper CS. Fusion of SYT to two genes, SSX1 and SSX2, encoding proteins with homology to the Kruppel-associated box in human synovial sarcoma. EMBO J. 1995; 14:2333-40.
8. Skytting B, Nilsson G, Brodin B, Xie Y, Lundeberg J, Uhlen M, Larsson O. A novel fusion gene, SYT-SSX4, in synovial sarcoma. J Natl Cancer Inst. 1999; 91:974-5.

9. Nielsen TO, Poulin NM, Ladanyi M. Synovial sarcoma: recent discoveries as a roadmap to new avenues for therapy. Cancer Discov. 2015; 5:124-34. https://doi. org/10.1158/2159-8290.cd-14-1246.

10. Panagopoulos I, Mertens F, Isaksson M, Limon J, Gustafson P, Skytting B, Akerman M, Sciot R, Dal Cin P, Samson I, Iliszko M, Ryoe J, Debiec-Rychter M, et al. Clinical impact of molecular and cytogenetic findings in synovial sarcoma. Genes Chromosomes Cancer. 2001; 31:362-72. https://doi. org/10.1002/gcc.1155.

11. Joseph CG, Hwang H, Jiao Y, Wood LD, Kinde I, Wu J, Mandahl N, Luo J, Hruban RH, Diaz LA Jr, He TC, Vogelstein B, Kinzler KW, et al. Exomic analysis of myxoid liposarcomas, synovial sarcomas, and osteosarcomas. Genes Chromosomes Cancer. 2014; 53:15-24. https://doi. org/10.1002/gcc.22114.

12. Carmody Soni EE, Schlottman S, Erkizan HV, Uren A, Toretsky JA. Loss of SS18-SSX1 inhibits viability and induces apoptosis in synovial sarcoma. Clin Orthop Relat Res. 2014; 472:874-82. https://doi.org/10.1007/ s11999-013-3065-9.

13. Haldar M, Hancock JD, Coffin CM, Lessnick SL, Capecchi MR. A conditional mouse model of synovial sarcoma: insights into a myogenic origin. Cancer Cell. 2007; 11: 375-88. https://doi.org/10.1016/j.ccr.2007.01.016.

14. Arnold MA, Arnold CA, Li G, Chae U, El-Etriby R, Lee CC, Tsokos M. A unique pattern of INI1 immunohistochemistry distinguishes synovial sarcoma from its histologic mimics. Hum Pathol. 2013; 44:881-7. https://doi.org/10.1016/j. humpath.2012.08.014.

15. Rekhi B, Vogel U. Utility of characteristic 'Weak to Absent' INI1/SMARCB1/BAF47 expression in diagnosis of synovial sarcomas. APMIS. 2015; 123:618-28. https:// doi.org/10.1111/apm.12395.

16. Iura K, Maekawa A, Kohashi K, Ishii T, Bekki H, Otsuka H, Yamada Y, Yamamoto H, Harimaya K, Iwamoto Y, Oda Y. Cancer-testis antigen expression in synovial sarcoma: NY-ESO-1, PRAME, MAGEA4, and MAGEA1. Hum Pathol. 2017; 61:130-9. https://doi.org/10.1016/j. humpath.2016.12.006.

17. Takenaka S, Naka N, Araki N, Hashimoto N, Ueda T, Yoshioka K, Yoshikawa H, Itoh K. Downregulation of SS18-SSX1 expression in synovial sarcoma by small interfering RNA enhances the focal adhesion pathway and inhibits anchorage-independent growth in vitro and tumor growth in vivo. Int J Oncol. 2010; 36:823-31.

18. Nagai M, Tanaka S, Tsuda M, Endo S, Kato H, Sonobe H, Minami A, Hiraga H, Nishihara H, Sawa H, Nagashima $\mathrm{K}$. Analysis of transforming activity of human synovial sarcoma-associated chimeric protein SYT-SSX1 bound to chromatin remodeling factor hBRM/hSNF2 alpha. 
Proc Natl Acad Sci U S A. 2001; 98:3843-8. https://doi. org/10.1073/pnas.061036798.

19. dos Santos NR, de Bruijn DR, Kater-Baats E, Otte AP, van Kessel AG. Delineation of the protein domains responsible for SYT, SSX, and SYT-SSX nuclear localization. Exp Cell Res. 2000; 256:192-202. https://doi.org/10.1006/ excr.2000.4813.

20. Saito T, Nagai M, Ladanyi M. SYT-SSX1 and SYT-SSX2 interfere with repression of E-cadherin by snail and slug: a potential mechanism for aberrant mesenchymal to epithelial transition in human synovial sarcoma. Cancer Res. 2006; 66:6919-27. https://doi.org/10.1158/0008-5472. can-05-3697.

21. Shui W, Gilmore SA, Sheu L, Liu J, Keasling JD, Bertozzi CR. Quantitative proteomic profiling of host-pathogen interactions: the macrophage response to Mycobacterium tuberculosis lipids. J Proteome Res. 2009; 8:282-9. https:// doi.org/10.1021/pr800422e.

22. Suehara Y, Tochigi N, Kubota D, Kikuta K, Nakayama R, Seki K, Yoshida A, Ichikawa H, Hasegawa T, Kaneko K, Chuman H, Beppu Y, Kawai A, et al. Secernin-1 as a novel prognostic biomarker candidate of synovial sarcoma revealed by proteomics. J Proteomics. 2011; 74:829-42. https://doi.org/10.1016/j.jprot.2011.02.033.

23. Robin YM, Penel N, Perot G, Neuville A, Velasco V, Ranchere-Vince D, Terrier P, Coindre JM. Transgelin is a novel marker of smooth muscle differentiation that improves diagnostic accuracy of leiomyosarcomas: a comparative immunohistochemical reappraisal of myogenic markers in 900 soft tissue tumors. Mod Pathol. 2013; 26:502-10. https://doi.org/10.1038/modpathol.2012.192.

24. Suehara Y, Kondo T, Fujii K, Hasegawa T, Kawai A, Seki K, Beppu Y, Nishimura T, Kurosawa H, Hirohashi S. Proteomic signatures corresponding to histological classification and grading of soft-tissue sarcomas. Proteomics. 2006; 6:44029. https://doi.org/10.1002/pmic.200600196.

25. Kawai A, Kondo T, Suehara Y, Kikuta K, Hirohashi S. Global protein-expression analysis of bone and soft tissue sarcomas. Clin Orthop Relat Res. 2008; 466:2099-106. https://doi.org/10.1007/s11999-008-0330-4.

26. El Beaino M, Araujo DM, Lazar AJ, Lin PP. Synovial Sarcoma: Advances in Diagnosis and Treatment Identification of New Biologic Targets to Improve Multimodal Therapy. Ann Surg Oncol. 2017; 24:2145-54. https://doi.org/10.1245/s10434-017-5855-x.

27. Spurrell EL, Fisher C, Thomas JM, Judson IR. Prognostic factors in advanced synovial sarcoma: an analysis of 104 patients treated at the Royal Marsden Hospital. Ann Oncol. 2005; 16:437-44. https://doi.org/10.1093/annonc/mdi082.

28. Machen SK, Easley KA, Goldblum JR. Synovial sarcoma of the extremities: a clinicopathologic study of 34 cases, including semi-quantitative analysis of spindled, epithelial, and poorly differentiated areas. Am J Surg Pathol. 1999; 23:268-75.
29. Kato H, Tjernberg A, Zhang W, Krutchinsky AN, An W, Takeuchi T, Ohtsuki Y, Sugano S, de Bruijn DR, Chait BT, Roeder RG. SYT associates with human SNF/SWI complexes and the C-terminal region of its fusion partner SSX1 targets histones. J Biol Chem. 2002; 277:5498-505. https://doi.org/10.1074/jbc.M108702200.

30. Naka N, Takenaka S, Araki N, Miwa T, Hashimoto N, Yoshioka K, Joyama S, Hamada K, Tsukamoto Y, Tomita Y, Ueda T, Yoshikawa H, Itoh K. Synovial sarcoma is a stem cell malignancy. Stem Cells. 2010; 28:1119-31. https://doi. org/10.1002/stem.452.

31. Zollner SK, Rossig C, Toretsky JA. Synovial sarcoma is a gateway to the role of chromatin remodeling in cancer. Cancer Metastasis Rev. 2015; 34:417-28. https://doi. org/10.1007/s10555-015-9575-z.

32. Garcia CB, Shaffer CM, Alfaro MP, Smith AL, Sun J, Zhao Z, Young PP, VanSaun MN, Eid JE. Reprogramming of mesenchymal stem cells by the synovial sarcomaassociated oncogene SYT-SSX2. Oncogene. 2012; 31:2323-34. https://doi.org/10.1038/onc.2011.418.

33. Hayakawa K, Ikeya M, Fukuta M, Woltjen K, Tamaki S, Takahara N, Kato T Jr, Sato S, Otsuka T, Toguchida J. Identification of target genes of synovial sarcomaassociated fusion oncoprotein using human pluripotent stem cells. Biochem Biophys Res Commun. 2013; 432:713-9. https://doi.org/10.1016/j.bbrc.2013.01.003.

34. Haldar M, Hedberg ML, Hockin MF, Capecchi MR. A CreER-based random induction strategy for modeling translocation-associated sarcomas in mice. Cancer Res. 2009; 69:3657-64. https://doi.org/10.1158/0008-5472. can-08-4127.

35. Ishida M, Miyamoto M, Naitoh S, Tatsuda D, Hasegawa T, Nemoto T, Yokozeki H, Nishioka K, Matsukage A, Ohki M, Ohta T. The SYT-SSX fusion protein down-regulates the cell proliferation regulator COM1 in $\mathrm{t}(\mathrm{x} ; 18)$ synovial sarcoma. Mol Cell Biol. 2007; 27:1348-55. https://doi. org/10.1128/mcb.00658-06.

36. Hsu KS, Kao HY. Alpha-actinin 4 and tumorigenesis of breast cancer. Vitam Horm. 2013; 93:323-51. https://doi. org/10.1016/b978-0-12-416673-8.00005-8.

37. Araki N, Hatae T, Yamada T, Hirohashi S. Actinin-4 is preferentially involved in circular ruffling and macropinocytosis in mouse macrophages: analysis by fluorescence ratio imaging. J Cell Sci. 2000; 113:3329-40.

38. Honda K, Yamada T, Endo R, Ino Y, Gotoh M, Tsuda H, Yamada Y, Chiba H, Hirohashi S. Actinin-4, a novel actinbundling protein associated with cell motility and cancer invasion. J Cell Biol. 1998; 140:1383-93.

39. San Martin IA, Varela N, Gaete M, Villegas K, Osorio M, Tapia JC, Antonelli M, Mancilla EE, Pereira BP, Nathan SS, Lian JB, Stein JL, Stein GS, et al. Impaired cell cycle regulation of the osteoblast-related heterodimeric transcription factor Runx2-Cbfbeta in osteosarcoma cells. J Cell Physiol. 2009; 221:560-71. https://doi.org/10.1002/ jep.21894. 
40. Ud Din N, Hopkins A, Memon A, Ahmad Z, Ahmed R. Calcifying/ossifying synovial sarcoma: a clinicopathologic and molecular study of 5 cases. Indian J Pathol Microbiol. 2015; 58:55-8. https://doi.org/10.4103/0377-4929.151188.

41. Winnepenninckx V, De Vos R, Debiec-Rychter M, Samson I, Brys P, Hagemeijer A, Sciot R. Calcifying/ossifying synovial sarcoma shows $\mathrm{t}(\mathrm{X} ; 18)$ with SSX2 involvement and mitochondrial calcifications. Histopathology. 2001; 38:141-5.

42. Hisaoka M, Matsuyama A, Shimajiri S, Akiba J, Kusano H, Hiraoka K, Shoda T, Hashimoto H. Ossifying synovial sarcoma. Pathol Res Pract. 2009; 205:195-8. https://doi. org/10.1016/j.prp.2008.10.003.

43. Kadoch C, Crabtree GR. Reversible disruption of mSWI/ SNF (BAF) complexes by the SS18-SSX oncogenic fusion in synovial sarcoma. Cell. 2013; 153:71-85. https://doi. org/10.1016/j.cell.2013.02.036.

44. Middeljans E, Wan X, Jansen PW, Sharma V, Stunnenberg $\mathrm{HG}$, Logie C. SS18 together with animal-specific factors defines human BAF-type SWI/SNF complexes. PLoS One. 2012; 7:e33834. https://doi.org/10.1371/journal. pone. 0033834 .

45. Soulez M, Saurin AJ, Freemont PS, Knight JC. SSX and the synovial-sarcoma-specific chimaeric protein SYT-SSX co-localize with the human Polycomb group complex. Oncogene. 1999; 18:2739-46. https://doi.org/10.1038/ sj.onc. 1202613 .
46. van der Vlag J, Otte AP. Transcriptional repression mediated by the human polycomb-group protein EED involves histone deacetylation. Nat Genet. 1999; 23:474-8. https:// doi.org/10.1038/70602.

47. Furuyama T, Banerjee R, Breen TR, Harte PJ. SIR2 is required for polycomb silencing and is associated with an $\mathrm{E}(\mathrm{Z})$ histone methyltransferase complex. Curr Biol. 2004; 14:1812-21. https://doi.org/10.1016/j.cub.2004.09.060.

48. Tanabe Y, Suehara Y, Kohsaka S, Hayashi T, Akaike K, Mukaihara K, Kurihara T, Kim Y, Okubo T, Ishii M, Kazuno S, Kaneko K, Saito T. IRE1alpha-XBP1 inhibitors exerted anti-tumor activities in Ewing's sarcoma. Oncotarget. 2018; 9:14428-43. https://doi.org/10.18632/oncotarget.24467.

49. Akaike K, Suehara Y, Kohsaka S, Hayashi T, Tanabe Y, Kazuno S, Mukaihara K, Toda-Ishii M, Kurihara T, Kim Y, Okubo T, Hayashi Y, Takamochi K, et al. PPP2R1A regulated by PAX3/FOXO3 fusion contributes to the acquisition of aggressive behavior in PAX3/FOXO1-positive alveolar rhabdomyosarcoma. Oncotarget. 2018; 9:25206-25215. https://doi.org/10.18632/oncotarget.25392.

50. Mukaihara K, Suehara Y, Kohsaka S, Akaike K, Tanabe Y, Kubota D, Ishii M, Fujimura T, Kazuno S, Okubo T, Takagi T, Yao T, Kaneko K, et al. Protein Expression Profiling of Giant Cell Tumors of Bone Treated with Denosumab. PLoS One. 2016; 11:e0148401. https://doi.org/10.1371/journal. pone. 0148401 . 